\title{
Beneficios del sistema de información en una entidad bancaria que opera en red: el caso de ING Direct
}

\author{
Juan Ros García \\ María del Rosario Ros García \\ Universidad de Murcia (España)
}

\section{Resumen}

Este estudio pretende demostrar los múltiples beneficios de la información, y más concretamente de los sistemas de información, a través del ejemplo de una entidad bancaria que opera en red. La información tiene una doble vertiente porque es una materia prima y a su vez está presente en todas las materias primas de la empresa. La principal ventaja de la organización es saber hacer uso de ella de forma eficaz, ya que el valor de la información es incalculable. La información aporta sus técnicas de recopilación; son sus herramientas de gestión, junto con la tecnología, para dar a la empresa la materia prima del crecimiento progresivo, el conocimiento.

Palabras clave: Gestión de la información. Banco en línea. Gestión del conocimiento. Empresas competitivas. ING Direct.

\section{Abstract}

The multiple benefits of information and knowledge management are demonstrated in this case study on ING Direct, a financial company that operates on-line. In the banking sector, information is both raw material and result of most of the firm processes. The best competitive advantage of this company is a remarkable information operation, which results in a growing and better knowledge base.

Keywords: Information management. On-line banks. Competitive management. Knowledge management. ING Direct.

\section{Objetivos}

Mediante esta investigación se pretende demostrar las múltiples ventajas tanto de la información como de los sistemas de información a través del ejemplo de ING Direct, una entidad bancaria que opera en red, es decir, por teléfono y por Internet. Nuestro objetivo principal se centrará en poner de manifiesto todos los beneficios

Scire. $14: 1$ (en.-jun. 2008) 141-153. ISSN 1135-3716. 
de la información, desde la identificación, la valoración, la recopilación, el almacenamiento y la recuperación hasta la distribución, siguiendo para ello las pautas que nos da Joan Majó (1988, pp. 9-11). De este modo la ubicó como bien económico y la reflejó así:

Ha aparecido un nuevo factor de producción, la información; y su utilización permite con ventaja la sustitución de los factores clásicos. Una mejor tecnología de proceso (información sobre cómo hacer las cosas) permite ahorrar energía y materias primas. Una mejor logística (información sobre cómo combinar las cosas) permite utilizar menos capital. Una adecuada automatización (informática) sustituye mano de obra. A medida que se va introduciendo más información, e información más estructurada, en el proceso de producción se van consiguiendo mejores condiciones de competitividad y se van sustituyendo los otros factores de producción.

La rapidez con la que una sociedad se va adaptando a esta nueva situación es la clave de su éxito económico. El futuro desarrollo no depende de disponer de mayor cantidad de recursos energéticos, minerales o incluso financieros. El futuro depende de disponer de la información y de la tecnología suficientes.

El objetivo derivado es que, una vez demostrados los beneficios que comenta el investigador Joan Majó, pasamos a estudiar el sistema de información de ING Direct y sus estrategias de comunicación actuales. Partimos de una metodología sintética. En primer lugar veremos si la identificación, la valoración, la recopilación, el almacenamiento, la recuperación, la distribución y otras herramientas de la información crean un sistema de información definido. En segundo lugar estudiaremos el banco directo. De la conjugación de estos dos primeros puntos obtenemos el tercero, que no es otro que la transformación del sistema de información en un sistema de conocimiento, y el cuarto, que son las ventajas que consigue el banco directo gracias al sistema de conocimientos. Respecto a las fuentes utilizadas en este artículo, las podemos dividir en dos grandes grupos: primarias y secundarias.

Las fuentes primarias se encuentran muy presentes debido a la facilidad de consulta garantizada por la Red. La empresa holandesa ING Direct habla de forma clara de su actividad, sus estrategias, su vinculación con el público en general, su identidad corporativa, sus productos en red, su preocupación social, etcétera. Toda esta gran información viene avalada por ser de primera mano.

Esta empresa busca contactar con su público objetivo a través de la Red (con su herramienta denominada acceso a clientes), lo cual demuestra la importancia de la información especializada y de la colaboración solícita que busca. Al mismo tiempo, los demás medios de comunicación que integran la totalidad de grupos informativos bombardean diariamente con sus anuncios, actos de relaciones públicas. Es decir, la sociedad es un gran campo de ensayo para las distintas informaciones de la empresa ING Direct, cuyo fin fundamental es el lucro. Además, publica gran cantidad de informativos para distribuir en los medios de comunicación, donde encuentra solamente a su público objetivo. Internet ofrece a la vez una gran para- 
doja, que es ser un medio de comunicación polifacético donde están presentes otros medios de comunicación que funcionan en red. Es una especie de matriz de medios, empresas, negocios (de fuentes), donde es posible documentarse con conocimientos de primera mano.

En este trabajo de investigación se estudian la empresa del sector secundario ING Direct y su sucursal bancaria en red (su página web), cuyo principal fin es ser cada día más rentable y por lo tanto estar más informada; por eso resaltamos la importancia de la Red como contacto directo con gran variedad de compañías que intentan conocer a las personas que navegan por ella para saber si su relación y su interés por la empresa se establecen por ser consumidores de sus productos. Incluso hay empresas, como es nuestro caso de estudio, que nacen y viven solo en la Red, y por lo tanto es este el único medio disponible para conocerlas y ver cómo desarrollan su actividad y cómo logran un nivel de beneficios tan interesante. También la Red es relevante para informarse sobre las relaciones de la empresa con otros colaboradores de esta; sin ella no podríamos conocer tan a la perfección estas relaciones tan baratas y efectivas a la vez. Hay veces en que se torna difícil conocer cómo se desarrollan algunas colaboraciones en red debido a claves de acceso (los clientes de ING Direct) y páginas exclusivas, que suponen un ahorro en comunicaciones patente para sus operarios y permiten ganar un tiempo significativo.

Las empresas, a su vez, desarrollan páginas web interesantes para dialogar permanentemente y de forma privada con sus clientes (acceso clientes), para conocer sus defectos y virtudes y avanzar hacía una rentabilidad plena.

Las fuentes secundarias, que se derivan de las primarias, son sobre todo bibliografía y se utilizan desde el punto de vista informativo. Destacamos la gran variedad de autores: unos representados por su gran avance investigador y otros, aunque no de tanta trascendencia en el mundo científico, por ser creadores de redes informatizadas. Desde el completo Manual de ciencias de la documentación, cuyo coordinador, José López Yepes, se introduce de forma clara y concreta cómo la información afecta a todas las áreas del conocimiento de las empresas públicas y privadas, y, sobre todo, se explica cuáles son las principales tendencias que influyen tanto en la empresa pública como en la privada. De este mismo autor debe resaltarse también la información sobre el método, las fuentes y la investigación desarrolladas en la aventura de la investigación científica, esto es, documentarse sobre los pasos a seguir para convertir la hipótesis de estudio en un método científico.

El desarrollo de las sociedades modernas va paralelo a la influencia que sus gobernantes ejercen, ya que sus legislaciones, sus incentivos, dificultan o facilitan la información tanto pública como privada. Este aspecto ha sido estudiado por la profesora Celia Chaín (2000) y el profesor Juan Ros García en el ámbito nacional y en el internacional, ya que en el avance de un país no solo influye la política nacional: también le afectan las legislaciones internacionales, debido a que la sociedad 
está cada vez más globalizada; hay una mayor especialización del trabajo, pero en el ámbito mundial.

Desde el punto de vista empresarial se imponen tendencias tales como la administración de la información de Fernando Ramos Simón, que habla de la unión de la información con la tecnología y de los beneficios obtenidos de la misma por las empresas.

\section{Análisis de los sistemas de gestión de la información y del conocimiento: metodología}

La información y el banco ING Direct necesitan un desarrollo metodológico desde cuatro puntos de vista.

\subsection{Las herramientas de la información crean un sistema de información (SI)}

La información es una comunicación o adquisición de conocimientos que permitan ampliar o precisar los que se poseen sobre una materia determinada.

Como dijimos en trabajos anteriores, la información tiene un valor fundamental, al igual que el dinero, los bienes de capital, el trabajo o materias como el método de identificación, la valoración, la recopilación, el almacenamiento, la recuperación, la distribución y el intercambio con otros recursos. La información recolectada puede ser transformada en resultados útiles, beneficiosos para conseguir los fines de la organización. Es un gasto cuyos costes pueden ser calculados con técnicas contables, lo que se verifica en este estudio sobre el banco electrónico. Presenta diversas alternativas de desarrollo y, aunque resulta difícil cuantificar el valor de esta información, su valía dependerá del uso que se le vaya a dar.

La información se encuentra presente en todos los niveles de actividad y en todas las ramas de la economía y de la política. Ningún responsable de una organización, del tipo que sea, toma hoy una decisión importante sin antes haber consultado una docena de informes. (Ramos, 1995, p. 58)

La información tiene una doble vertiente porque es una materia prima y a su vez está presente en todas las materias primas de la empresa. La principal ventaja de una organización es saber hacer uso de ella de forma eficaz, ya que el valor de la información es incalculable. La información aporta sus técnicas de recopilación; son sus herramientas de gestión, junto con la tecnología, para dar a la empresa la materia prima del crecimiento progresivo, el conocimiento.

En el caso del banco en red ING Direct, la información, su recopilación, su gestión y su distribución pasan a ser la guía para la solución de situaciones nuevas; los beneficios de trabajar con información, sus estrategias, la utilidad de su recopilación y las tecnologías para crear un sistema de información son necesarios para su funcionalidad. Por tanto, este es el objeto de estudio: conocer el valor de la infor- 
mación se centra en aprovechar sus ventajas como materia prima cuyo gen sanea unos negocios y crea otros.

Como ya dijimos en estudios anteriores (Ros, 2006), un sistema de información sería lo más parecido al sistema térmico de una habitación, que quizá sea difícil de definir y descubrir, pero cuyos efectos son palpables:

Sabemos cómo comportarnos, abrir o cerrar ventanas, subir o bajar el termostato, adivinamos que en el interior existe un sistema inteligente y sabemos lo cómodo que es adquirir un servicio llave en mano. Sabemos también que subyacen determinados oficios: ingenieros, arquitectos, decoradores, obreros, electricistas, calefactores, etc., que han hecho posible tal diseño, tal sistema.

Toda empresa es un sistema de información. Toda materia prima, departamento, personal, clientes... contienen una información, y su aprendizaje es vital para el ciclo de vida de la empresa, para conseguir que perdure a pesar de la feroz competencia. Tener información significa reducir los gastos considerablemente, porque evita cometer errores y estos tienen un coste muy grande. Incentivar la recolección de la información relevante para la entidad significa también reforzarla, debido a la comunicación que se establece de forma mecánica entre todos sus usuarios. Así, podemos afirmar que la recolección de los hechos, de los datos, crea informaciones que permiten obtener conocimientos interesantes para incrementar el desarrollo y reducir los despilfarros. Utilizamos otra definición de sistema de información:

La actividad documentaria se concreta en los sistemas de información y Documentación. Estos son conjuntos de personas, máquinas y procedimientos que transforman una información de entrada en una información de salida susceptible de ser utilizada como base para la obtención de una nueva información. (López Yepes, 2002, p. 55)

El sistema de información da vida en la empresa, crea actualidad en informaciones obsoletas, es el encargado de dar una nueva apertura a la información y, por contagio, a la empresa. Estos flujos de información se transforman en flujos de crecimiento.

\subsection{El banco directo}

Si analizamos el término banco directo vemos que se refiere a un banco con un funcionamiento en tiempo presente, ya que se gestiona directamente por el usuario a través de Internet o por medio del teléfono. En definitiva, es una sucursal bancaria disponible en el ordenador personal de cada usuario, con un acceso para clientes que sirve de servicio llave en mano para operar y resolver las posibles necesidades bancarias en el presente, a cualquier hora y cualquier día. Antes de analizar su principal ventaja frente a los bancos tradicionales vamos a centrarnos en contextualizar este banco.

ING Direct pertenece al grupo financiero holandés ING, cuya área de negocio más conocida en España es la aseguradora Nationale-Nederlanden; concretamente Scire. $14: 1$ (en.-jun. 2008) 141-153. ISSN 1135-3716. 
pertenece a su negocio estratégico denominado banca directa. Comenzó a operar en España el 12 de mayo de 1999, y este es el primer país europeo donde ING Direct trabaja al margen de Holanda. Es decir, nuestro país ha servido de campo de ensayo para este novedoso proyecto bancario, que después se ha extendido con éxito a Francia, Italia, Alemania y Reino Unido.

Comenzamos ahora a aplicar de forma práctica las ventajas que atribuye Joan Majó a la información, como es el hecho de permitir la sustitución de factores clásicos. En el caso de ING Direct, se han reemplazado las sucursales bancarias por una página web y los banqueros por teleoperadoras; es decir, la información ha reducido sustancialmente sus costes fijos.

Continuamos con los beneficios que atribuye Joan Majó a la información: una mejor tecnología de procesos (información sobre cómo hacer las cosas) permite ahorrar energía y materias primas. El banco en red permite un ahorro de electricidad y de trabajo, ya que los propios clientes son empleados del banco porque ellos mismos realizan sus operaciones bancarias con sus ordenadores personales; es decir, el cliente pone incluso su propio ordenador al servicio de la empresa, mientras que en un banco convencional es la entidad la que soporta los costes de compra y mantenimiento de material informático.

En un banco convencional las operaciones bancarias se canalizan a través de sus empleados, los cuales suponen un gran coste adicional, con gastos tales como cuotas de la seguridad social, vacaciones y bajas, aunque hay bancos que saben sacar el máximo rendimiento a sus trabajadores, por el trato personalizado que ofrecen a los clientes y por utilizarlos como fuentes de información de primera mano.

Además, los bancos convencionales soportan gastos de cada una de sus sucursales: facturas de luz, teléfono, agua, contribución y otros gastos asociados a su mantenimiento, como seguros o costes de modernización, desde cámaras de vigilancia y cámaras acorazadas hasta cajeros automáticos, etcétera.

En resumen, el banco directo ING Direct, perteneciente al grupo holandés ING, al no disponer de sucursales, ahorra energía, luz, agua, contribución, gastos de modernización de sucursales, cámaras de vigilancia y acorazadas y cajeros automáticos; presta un mejor servicio, sin esperas innecesarias o discusiones con empleados poco eficaces, y resuelve los problemas en el momento que quiera el cliente; dispone de una mejor logística y, debido a que trabaja en línea, ahorra costes tanto cuando extrae o abona en las cuentas de otras entidades bancarias como cuando obtiene información puntual y exacta de las necesidades del cliente; utiliza menos capital, ya que no abona alquileres de sucursales ni material de oficina ni seguros empresariales para cada local, sino que los sustituye por una sede central en la localidad madrileña de Las Rozas y dos oficinas de apoyo, una en Barcelona (en el número 538 de la avenida Diagonal) y otra en Madrid (en el 34 de la 
calle José Ortega y Gasset); prescinde de mano de obra, al cambiar una gran plantilla de gestores bancarios por teleoperadoras y un cuadro directivo compuesto por ocho personas: Miguel Crespo (secretario general), Carmen de Medraño (recursos humanos), Miguel San Pablo (operaciones y tecnología), Alfonso Zapata (dirección financiera), Juan Manuel Granados (cuenta naranja), Carina Szpilka (hipoteca naranja), Javier Antonio (productos de inversión) y Weber Zippold (nuevos proyectos).

En definitiva, ING Direct ha cambiado los factores de producción clásicos del sector de la banca por la información y la tecnología.

\subsection{Transformación del sistema de información en un sistema de conocimiento}

El uso del sistema de información lleva a la empresa a buscar la especialización, y de ahí surge la calidad como fuerte ahorro de procesos. La calidad debe primar en una organización, pues sirve de filtro limpiador. Si prescindimos de ella estamos utilizando de forma errónea los recursos de una organización, manteniendo sectores inservibles de la misma. Y cuando hablamos de información necesitamos un filtro más espeso, un depurador. El SI es un valor para la empresa, ya que facilita enormente la identificación, la valoración, la recopilación, el almacenamiento, la recuperación y la distribución de la información. Así, la tecnología es la herramienta fundamental para trasmitir conocimientos (Chaín, 2000, p. 199):

La nueva aplicación de la filosofía de gestión de calidad a los sistemas de información se asocia a la confluencia de tres factores: la aplicación de la teoría sistemática a los servicios de información, que facilita la definición de modelos dinámicos de organización, la implantación entre los profesionales de una nueva "filosofía de servicio" y la adopción de un cierto concepto empresarial y de técnicas moderna de gestión.

El sistema de información contiene un resumen de todos los temas que le son de interés, su historia, su futuro. Su uso es el que permite sacar conclusiones a la empresa sobre su filosofía de actuación. La funcionalidad de este SI, su control y su estudio lo llevarán a mudar en un sistema de conocimiento:

Cada vez más, el futuro de una empresa depende del conocimiento sobre patentes, procesos, capacidad de gestión, tecnologías, información sobre clientes y proveedores, experiencia tradicional; la suma de todo esto es la sabiduría, constituye el capital intelectual corporativo. (Ibídem)

Un sistema parecido a Internet pero en la empresa permitiría consultar todos los informes que un directivo necesitara en el acto y tomar la decisión adecuada. Una vez clara la solución, debería emplear la estrategia de mercado más apropiada para resolver el problema. Este sistema debería recoger los conocimientos de la empresa, pero tendría que disponer también de la información del mercado. Una empresa no está formada solamente por sí misma; el mercado, los consumidores, 
los distribuidores, la competencia, los proveedores y la sociedad son parte de ella, y de todo este conjunto depende su futuro. De este modo, de la calidad del trabajo de estos grupos depende el futuro de la empresa.

El conocimiento, la tecnología y la empresa se hacen rentables cuando satisfacen plenamente las necesidades de los clientes, cuando se adelantan a satisfacer los futuros problemas de los usuarios. Las empresas necesitan moverse de forma humana para conocer y responder a los problemas de los clientes.

Para mejorar la calidad de vida y el bienestar de los usuarios se necesita información sobre ellos, es decir, un sistema de información. Primero hay que identificar quiénes son las personas que utilizan los servicios de la empresa pública o privada: cliente final, sociedad, empleado, distribuidor y proveedor. Una vez detectados los grupos de personas que usan los servicios, tenemos que recopilar datos relevantes sobre ellos para luego convertirlos en conocimientos. Una manera sencilla de obtener dicha información es concienciar a usuarios y empresas de que es vital para el crecimiento de estas la conexión vía red con ellos, es decir, un sistema de conocimientos, el cual facilitará para todos una gran cantidad de conocimientos que les llevarán a resolver cuestiones comunes con solo pulsar una tecla. Este es el primer principio de desarrollo de una comunidad: el reconocimiento por parte de los implicados en ella de que necesitan trabajar en equipo para obtener mayores beneficios y disminuir el número de problemas.

El conocimiento y la tecnología deben aunar sus esfuerzos para concienciar al conjunto de quienes participan en este ciclo de que su crecimiento es una misión común para todos. Para mejorar el nivel de vida de una empresa y su comunidad no hay más secreto que preguntar sobre los problemas que dificultan el día a día y darles una solución rápida y eficaz entre todos los implicados, ya que ellos son los que conocen sus carencias y soluciones. En definitiva, conocer es saber lo que ocurre en nuestro entorno y movernos a su ritmo. La empresa necesita como materia para desarrollarse conocimientos oportunos que le permitan resolver sus problemas de forma mecánica.

Las empresas, en todos los niveles de actividad y en todas las ramas de la economía y de la política, no pueden ser una coraza inaccesible para la sociedad, los proveedores, etcétera; deben ser un sistema abierto (un sistema de conocimientos) de interacción para todos los implicados en su economía con el fin de extraer las ventajas que dan sus informaciones. De acuerdo que no hay que contentar a todo el mundo, pero sí a quienes tienen autoridad para opinar sobre la empresa. El análisis de situación de una empresa define muy bien a todos los implicados en su crecimiento (Ros y Cayero, 2003, p. 58):

La gestión de la información es una respuesta articulada para el desarrollo de factores interrelacionados, como los siguientes: a) el continuo crecimiento del volumen y variedad de la información documentada; b) la convergencia de las tecnologías asocia- 
das a la creación, comunicación y diseminación de la información; c) la importancia cada vez mayor de la información como recurso clave de las organizaciones; d) la necesidad de gestionar este nuevo recurso de modo más eficaz.

Como dijimos en otras ocasiones, el sistema de información cambia al sistema de conocimientos cuando son los propios empleados de la empresa los principales interesados en disponer de un sistema de mejora continua que solucione sus dificultades en el presente.

Hay compañías públicas y privadas que mantienen relaciones incluso con sus competidores para diversificarse y crecer todos de forma productiva. Gestionar conlleva tener una mente abierta, conocer lo que sucede en el mercado, y para eso debemos analizar de forma pormenorizada lo que ocurre en el interior de la firma: si se cosecha la información, si las nuevas tecnologías transmiten los conocimientos, si estos se comunican con efectividad y si el conocimiento es la clave de gestión de la empresa. En definitiva, es necesario gestionar el nuevo recurso de forma eficaz. Si paramos en este recurso interno conoceremos una cantidad de oportunidades significativas. Actualmente la gestión es la clave del desarrollo empresarial, pero hablamos de gestión como sinónimo de motivación: dirigir para que cada empleado de la organización dé lo mejor de sí; de esta manera, una firma en la que cada miembro posee la motivación y la formación adecuadas obtiene los mejores resultados, como son el espíritu de mejora continua, de hacer el trabajo de la forma más productiva y empleando el menor tiempo posible. Como hemos señalado con anterioridad, una empresa dispone de un sistema de conocimientos cuando son los propios trabajadores quienes los buscan; es decir, los empleados se convierten en el motor de recuperación de conocimientos. Estos se dan cuenta de la importancia del conocimiento como recurso clave para disminuir sus horas laborales y aumentar su productividad.

Cada área de negocio representa un grupo de personas que trabajan por las alianzas estratégicas, que son fundamentales para que la información se reduzca al conocimiento y las tecnologías creen una comunicación productiva y lleven a cualquier institución, sea pública o privada, a ser líder en su campo.

\subsection{Ventajas del banco directo gracias al sistema de conocimientos}

A medida que se introduce más información en el proceso de producción se consiguen mejores condiciones de competitividad y se van sustituyendo los otros factores de producción.

Podemos afirmar que ING Direct es un gran sistema de conocimientos: informa a los clientes de cómo hacer las cosas porque ofrece conocimientos especializados sobre cómo fluctúa el sector de la banca. Por tanto, liberaliza el sector, y además trata sus ingresos como activos de inversión seguros gracias a su sistema de conocimientos, ofrece un servicio inmediato en el propio ordenador del usuario, lo 
que evita colas e incluso empleados poco efectivos. Este novedoso sistema informa a sus usuarios de las últimas tecnologías en comunicación.

Es fundamental el paso de la concepción de la información como servicio a la de recurso económico, a la de bien informativo. En efecto la información es un bien, bien capaz de generar un valor determinado cuando aumenta su papel en aquello que puede afectar a la empresa y cuando incide en la acertada toma de decisiones. (López Yepes, 2002, p. 189)

La estrategia del banco ING Direct es sólida y diferenciadora. Consiste en que el cliente es el propio diseñador del producto gracias a un ciclo comunicativo muy fluido donde el emisor es el cliente (y emite en el momento que a él le viene bien, es decir, cuando quiera conectarse con su ordenador), el receptor es la empresa, el canal es Internet o el teléfono y el mensaje son sus necesidades reales. Los mensajes de cada cliente son archivados en este sistema de conocimientos y su agrupación representa las preferencias generales de los usuarios y la base para establecer una mejora continua de sus productos al ritmo que le marque el mercado.

La utilización de Internet y el teléfono, así como la plantilla ajustada, permite que los costes solo supongan el 0,35\% del pasivo, mientras que para los competidores es, de media, el 2,5\%. Nuestros gastos se comen muy poco de la rentabilidad que obtenemos colocando el pasivo de los clientes. A ellos les cedemos gran parte de nuestro beneficio. Esa es la fórmula del éxito: si tú le das parte de lo que ganas al cliente, él te lo devuelve por otras vías. Nuestro éxito es la suma de muchos pocos. (El País [en línea], 30 de mayo de 2004, p. 74)

El precio es la rentabilidad, es decir, el producto es más rentable para el cliente debido a los escasos costes fijos que soporta la entidad y que son invertidos en un sistema de mejora continua de sus servicios; el cliente devuelve esta prestación contratando nuevos productos y recomendando la entidad a nuevos clientes. El canal de distribución es exclusivo para cada cliente al tratarse de servicios llave en mano con una contraseña, que corresponde al NIF, pasaporte o tarjeta de residencia del cliente, y una clave de usuario representada por la fecha de nacimiento de este. El canal de distribución es propiedad de ING Direct y conecta con el cliente directamente, sin intermediarios; por lo tanto, se evitan malentendidos y mala gestión por parte de terceros, de modo que se gana en una estrategia de comunicación personalizada a través de la Red, e incluso, como hemos dicho, los clientes convencidos son los principales perceptores de venta y los protagonistas de las campañas publicitarias en los medios above the line (los medios tradicionales, como son radio, televisión y prensa).

La innovación tecnológica es la principal arma competitiva de la empresa moderna. La tecnología que una empresa necesita puede generarla internamente o adquirirla en el exterior, pagando por ella el precio correspondiente, bien sea a otras empresas o de la competencia o a instituciones públicas o privadas especializadas en la investigación y desarrollo tecnológico. (Ramos, 1995, p. 125) 
Fruto de la excelencia en la combinación de las estrategias de conocimientos es un sector de negocio (banco directo) tan efectivo que, como hemos afirmado, convierte a sus clientes en perceptores de venta, es decir, estos transmiten las ventajas a sus conocidos, realidad que se refleja en las campañas actuales en los principales medios de comunicación, y además ofrecen un servicio espléndido. Su motivación de venta consiste en ser asesores personalizados y gratuitos para cada ciudadano; tratan cada ingreso como un activo de inversión seguro (ya que no solo la sucursal soporta bajos costes: sus clientes, también). Todas estas ventajas hacen que la compañía ING Direct sea poseedora de una imagen de marca impecable, que suple a la perfección la ausencia de sucursales.

El estudio del negocio desarrollado por el banco en línea ING Direct demuestra de forma sencilla la utilidad de la información y las herramientas del SI.

En la enumeración de las ventajas de la información por parte de Joan Majó, nos detenemos en el punto que dice que una mejor logística (información sobre cómo combinar las cosas) permite utilizar menos capital. El ejemplo de este banco nos demuestra que su logística en línea permite ahorrar costes. ING trabaja extrayendo o abonando en las cuentas de otras entidades bancarias lo que desea el cliente, y también se apoya en el teléfono y en el correo convencional; es decir, su logística se reduce a diversas informaciones en red con un coste muy bajo tanto en tiempo como en material. Este intercambio de información extraída directamente de sus fuentes (los clientes, mediadores bancarios, teleoperadoras) le ha proporcionado los conocimientos suficientes para haber adquirido en estos seis años un saber hacer interesante que le otorga ventajas frente a su competencia. Majó afirma también que una adecuada automatización (informática) sustituye mano de obra, como ya hemos comentado en este caso particular: suple una gran plantilla de gestores bancarios por teleoperadoras y muchas sucursales por una sola sede central.

A medida que ING Direct introduce más información, o sea, más datos sobre sus clientes, sus necesidades, el mercado, etcétera, ofrece productos más ajustados a su demanda y por tanto mejora su competitividad y sustituye factores de producción clásicos como sucursales, electricidad, teléfono, ordenadores, material de oficina, salarios o plantilla e invierte lo ahorrado en procesos de mejora continua, es decir, su sistema de información ahorra costes y extrae beneficios cuantiosos para todos sus clientes y socios.

La rapidez con la que el grupo holandés inició este negocio digital en España y posteriormente en Francia, Italia, Alemania, Estados Unidos y Reino Unido es una de las claves de su éxito. Sus conocimientos sobre el saber hacer en banca digital desde 1999, junto con la liberalización de un sector tan clásico como es el bancario, le han permitido ofrecer a sus posibles clientes información especializada sobre los distintos productos financieros, así como unas mejores condiciones para negociar con sus bancos de siempre o para convertirse en usuarios de esta entidad Scire. $14: 1$ (en.-jun. 2008) 141-153. ISSN 1135-3716. 
en red. De este modo informa sobre distintos productos como son la hipoteca naranja, el depósito naranja a un 6\% TAE, la cuenta naranja, los fondos de inversión, los planes de pensiones y el broker naranja.

En resumen, este sistema supone un ahorro considerable de electricidad, material de oficina, ordenadores y tiempo, tanto en la tarea de informar sobre el sector bancario (de forma clara y sencilla) como en la de gestionar los movimientos monetarios en red en tiempo presente, y además evita situaciones incómodas (colas en el banco, empleados poco efectivos, etcétera).

ING Direct ha puesto a disposición de los clientes que quieran contratar un préstamo hipotecario un asesor personal especializado que se encargará de guiarle durante todo el proceso de tramitación de la hipoteca y resolverá todas sus dudas. (La Gaceta de los Negocios [en línea], 28 de julio de 2004, p. 17)

Un ahorro de materias primas significa, en el caso del banco en red, un mejor servicio, ya que se puede atender a los clientes on-line justo cuando estos lo necesitan y ofrecerles asesores especializados.

\section{Resultados}

Se demuestra el objetivo principal del estudio. Así, podemos afirmar que una mejor tecnología de proceso permite ahorrar energía y materias primas: de este modo la entidad bancaria soporta menos costes fijos que la banca convencional; una mejor logística permite utilizar menos capital: su sistema de información es la Red, lo que hace que apenas tenga costes; una adecuada automatización sustituye mano de obra: la plantilla es mínima al no tener sucursales físicas.

Respecto a los objetivos derivados, podemos señalar que este sistema de conocimiento desarrolla estrategias de comunicación al instante y llega a un punto en el que sus usuarios son sus principales perceptores de venta.

\section{Conclusiones}

Verificamos que con los sistemas de información se consiguen mayores condiciones de competitividad y se van sustituyendo los otros factores de producción, además, el futuro depende de disponer de la información y de la tecnología suficientes.

\section{Recomendaciones}

Es conveniente que tanto las entidades públicas como las privadas dispongan de un sistema de información. El plan de la sociedad del conocimiento denominado España.es facilita ayuda a las pymes para su puesta en marcha. 


\section{Referencias}

Chaín Navarro, C. (2000). Gestión de información en las organizaciones. Murcia: DM, 2000.

Cinco Días. http://www.cincodias.com (2005-11-14).

Drucker, P. (1992). El ejecutivo eficaz. Barcelona: Edhasa, 1992.

El País. http://www.elpais.es (2004-05-30).

Expansión. http://www.expasión.es (2005-11-14).

ING Direct. http://www.ingdirect.es (2005-11-14).

Kotler, P. (2002). Dirección de marketing. Madrid: Prentice Hall, 2002.

La Gaceta de los Negocios. http://www.negocios.com/gaceta (2004-07-28).

López Yepes J. (1995). La aventura de la investigación científica: guía del investigador y del director de investigación. Madrid: Síntesis, 1995.

López Yepes, J. (coord.) (2002). Manual de ciencias de la documentación. Madrid: Pirámide, 2002.

Majó, J. (1988). Prólogo del libro. // Coll-Vicent, R. Información y poder. Barcelona: Heder, 1988. 9-11.

Ramos Simón, Luis Fernando (1995). Dirección, administración y marketing de empresas e instituciones documentales. Madrid: Síntesis, 1995.

Ramos Simón, Luis Fernando (2003). Introducción a la administración de información. Madrid: Síntesis, 2003.

Ros García, J. (1994). Documentación general. Sistemas, redes y centros. Madrid: Síntesis, 1994.

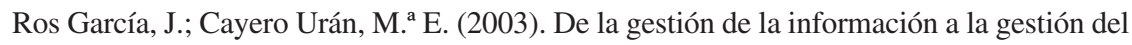
conocimiento. // Investigación Bibliotecológica. 17: 34 (2003) 54-69.

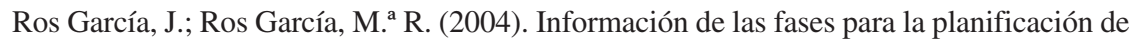
la rentabilidad empresarial. // Revista General de Información y Documentación. 14:1 (2004) 79-96.

Ros García, María de Rosario (2006). El gestor de la información, el gestor de marketing y el gestor del conocimiento. // Documentación de las Ciencias de la Información. 29. (2006) 279-299

Scire. $14: 1$ (en.-jun. 2008) 141-153. ISSN 1135-3716. 